\title{
Suture Slippage During Anterior Cruciate Ligament Graft Passage Is Significantly Lower Using a Krackow Suture
}

\author{
Tatsuo Mae, M.D., Ph.D., Kazunori Shimomura, M.D., Ph.D., Tomoki Ohori, M.D., Ph.D., \\ Takehiko Hirose, M.D., Shuji Taketomi, M.D., Ph.D., Tomoyuki Suzuki, M.D., Ph.D., and \\ Ken Nakata, M.D., Ph.D.
}

\begin{abstract}
Purpose: To compare the suture slippage on a hamstring tendon graft prepared with a modified finger-trap device (SPEEDTRAP) with one prepared with Krackow stitch during graft passage through the tibial tunnel in ACL reconstruction. Methods: Thirty-eight patients underwent anatomic triple-bundle anterior cruciate ligament reconstruction with 2 femoral and 3 tibial tunnels. After semitendinosus tendon was cut in half to make 2 grafts, the free ends of the proximal membranous portion (posterolateral [PL] graft) were prepared together with 2 sutures: (1) one SPEEDTRAP and one Krackow stitch for 20 cases (group A) and (2) double Krackow stitches on both sides for 18 cases (group B). Then, the PL graft was dye-marked at the proximal suture of SPEEDTRAP in group A and Krackow suture in group B and was inserted into the joint via tibial tunnel ahead of the loop side. The distance between the mark on the graft and the proximal suture of SPEEDTRAP or Krackow stitch was measured under arthroscopy after graft fixation at femur. Slippage was defined as $1 \mathrm{~mm}$ and more of distance between the mark and the proximal suture. Results: Slippage was observed in 16 cases for SPEEDTRAP and in 2 for Krackow suture in group A, whereas one case showed slippage in group B. The slippage distance was $4.0 \pm 2.9 \mathrm{~mm}$ for SPEEDTRAP and $0.2 \pm 0.5 \mathrm{~mm}$ for Krackow stitch in group A $(P<.001)$, whereas it was $0.1 \pm 0.2 \mathrm{~mm}$ for double Krackow stitch in group B, showing a significant difference from SPEEDTRAP suture $(P<.001)$. Conclusions: At the time of PL graft passage through the tibial tunnel in anterior cruciate ligament reconstruction, there was significantly less slippage observed with the Krackow stitch compared with the SPEEDTRAP stitch. Level of Evidence: Level IV, therapeutic case series.
\end{abstract}

$\mathbf{H}$ amstring tendon graft preparation is one of the basic and essential techniques in ligament reconstructions, and various suture techniques have been

From the Departments of Sports Medical Biomechanics (T.M.) and Orthopaedics Surgery (K.S., T.O., T.H.), Osaka University Graduate School of Medicine, Osaka; Department of Orthopaedic Surgery, The University of Tokyo, Tokyo (S.T.); Department of Orthopaedic Surgery, Sapporo Medical University School of Medicine, Sapporo (T.S.); and Department of Sports and Performing Arts, Osaka University Graduate School of Medicine, Osaka (K.N.), Japan.

The authors report that they have no conflicts of interest in the authorship and publication of this article. Full ICMJE author disclosure forms are available for this article online, as supplementary material.

Received January 27, 2021; accepted June 21, 2021.

Address correspondence to Tatsuo Mae, M.D., Ph.D., Department of Sports Medical Biomechanics, Osaka University Graduate School of Medicine, 2-2, Yamada-oka, Suita-city,Osaka, 565-0871, Japan.E-mail: ta-mae@umin.ac.jp

(C) 2021 THE AUTHORS. Published by Elsevier Inc. on behalf of the Arthroscopy Association of North America. This is an open access article under the CC BY-NC-ND license (http://creativecommons.org/licenses/by-nc-nd/4.0/).

2666-061X/21133

https://doi.org/10.1016/j.asmr.2021.06.003 reported. ${ }^{1-5}$ The Krackow stitch ${ }^{1}$ is the conventional technique for graft preparation and is widely performed because of high fixation strength. Sakaguchi et al. ${ }^{6}$ compared the biomechanical properties of porcine tendon fixation among 3 different common stitch methods and concluded that the Krackow stitch was superior to the baseball stitch and the whipstitch stitch in elongation at 1,500 loading cycles and maximum failure load. However, preparation of graft may damage a tendon, can be time-consuming, and has the risk of needle-stick injury, as most suture techniques require needle-repeated passage through a tendon. Furthermore, passing the needle and suture through the tendon can disrupt the tendon longitudinal fiber and can weaken the material properties of graft. ${ }^{7}$

To solve these disadvantages, different tendon grasping techniques have been reported. Su et al. ${ }^{3}$ proposed the modified finger-trap (MFT) suture technique, based on the principle of Chinese finger trap, while the suture was tied over the graft without needle stitching. Camarda et al. ${ }^{8}$ measured the time for graft 
preparation with the MFT suture and reported the MFT technique required approximately 30 seconds and could reduce the suture times compared with the time of the Krackow stitch and the Whipstitch. SPEEDTRAP (Depuy-Mitek, Raynham, MA) recently was released for suture-preparation of a soft-tissue graft without needles and could easily create a tuber tendon configuration based on the principle of Chinese finger trap. Barber et al. ${ }^{9}$ compared fixation security and biomechanical strength using bovine flexor tendon among the following different graft suture techniques: the Krackow stitch, the FiberLoop, the SPEEDTRAP, the WhipKnot, and loop-in-loop stitch. They reported that the SPEEDTRAP suture showed no damage at the tendon-suture interface and was equal in elongation to the other techniques after cyclic loading test, whereas it had the greatest maximum load to failure.

Our policy in the case of ligament reconstruction is that the tunnel diameter should be as small as possible to reduce wasted space and the graft must fit snugly in the tunnel. There are concerns that coarse-grained suture techniques or poor graft material causes some slippage between suture and tendon during graft passage through the tibial tunnel because of friction. However, few studies have focused on the slippage of sutures on graft during graft passage in ligament reconstruction, whereas the slippage of suture during graft passage would be clarified in this study. The purpose of this study was to compare the suture slippage on a hamstring tendon graft prepared with a modified finger-trap device (SPEEDTRAP) with one prepared with Krackow stitch during graft passage through the tibial tunnel in anterior cruciate ligament (ACL) reconstruction. Our hypothesis was that the slippage of the SPEEDTRAP suture would be larger than that of the Krackow suture on the graft.

\section{Methods}

Patients who underwent primary anatomic triplebundle ACL reconstruction with autogenous hamstring tendon grafts ${ }^{10}$ for ACL injury from March 2018 to March 2019 were retrospectively identified. Those with multiple ACL reconstruction or multiple ligament injuries were excluded. We collected demographic information and details regarding the injuries and repairs. This study protocol (\#17047) was accepted by our institutional review board.

\section{Surgical Procedure}

The resident's ridge and posterior cartilage margin could be clearly visualized as a landmark for the ACL footprint after removal of ACL remnant. Two 2.4-mm guidewires were separately inserted into the footprint from lateral femoral cortex with the anterolateral entry femoral aimer (Smith $\&$ Nephew Endoscopy, Andover, MA). Then, two 5.0- to 5.5-mm tunnels were created by overdrilling via the guidewires. For the tibia, three 2.4-mm guidewires were inserted in each portion of ACL attachment surrounded by anterior ridge, medial intercondylar ridge, and anterior horn of the lateral meniscus with the tibial tip aimer (Smith 8 Nephew Endoscopy) respectively. Then, 2 anteriorly located guidewires for the medial portion of anteromedial (AM-M) and the lateral portion of anteromedial (AM-L) grafts were overdrilled with $4.5-$ to $5.0-\mathrm{mm}$ drills, whereas the posteriorly located guide wire for the posterolateral (PL) graft was overdrilled with a $5.0-\mathrm{mm}$ drill, matching the grafts' diameters.

\section{Graft Preparation}

A semitendinosus tendon was harvested and was cut in half to prepare 2 double-looped tendon grafts. Two ENDOBUTTON-CLs (Smith \& Nephew Endoscopy) were connected to each loop end of the graft. The appropriate continuous loop length was determined on the basis of the femoral tunnel length to introduce sufficient graft materials of more than $13 \mathrm{~mm}$ into the femoral tunnels. A \#2 ULTRABRAID suture (Smith \& Nephew, Andover, MA) was placed on each free end of the distal tendinous portion in Krackow stitch fashion for 2-limb grafts (AM-M/-L graft). For the proximal membranous portion, its free ends were prepared together (1) with one SPEEDTRAP and one Krackow stitch using one \#2 ULTRABRAID suture for 20 cases from March 2018 to July 2018 (group A) and (2) with double Krackow stitches with two \#2 ULTRABRAID sutures for 18 cases from August 2018 to March 2019 (group B) to make one-limb graft (PL graft). In group A, a SPEEDTRAP suture was used after suture in Krackow stitch was completed on one side of folded graft. SPEEDTRAP of 20 $\mathrm{mm}$ in length was used and gripped graft by more than $20 \mathrm{~mm}$ from the free end. The PL graft was dye-marked at the proximal suture of SPEEDTRAP in group A and one Krackow suture in group B after preloading on the graft preparation table for approximately 3 minutes to achieve firm fixation with suture as well as to remove the creep of graft (Fig 1).

\section{Graft Fixation and Slippage Measurement}

The loop end of the PL graft was introduced through the tibial tunnel to the femoral tunnel and was fixed on the lateral femoral cortex by turning the ENDOBUTTON. The proximal suture of SPEEDTRAP or the Krackow stitch and the mark on the graft were arthroscopically took photos with a probe to measure the slipped distance just after flipping the ENDOBUTTON, as the location of the proximal suture on graft could be easily observed in the joint. The intraclass correlation coefficients was 0.97 in intrarater reliability. The ratio of cases with slippage also was assessed, whereas suture slippage was defined as the suture slipped by $1 \mathrm{~mm}$ and more. Then, the slippage of SPEEDTRAP was compared with that of Krackow suture 

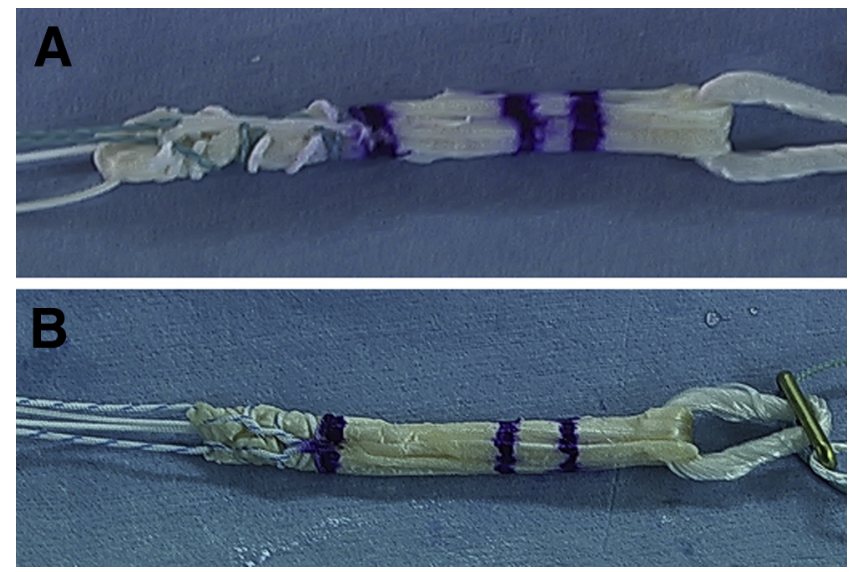

Fig 1. Prepared posterolateral graft with a dye mark at the proximal end of the suture. (A) One SPEEDTRAP suture (green/ white suture) and one Krackow suture (white suture). (B) Double Krackow sutures (white and white/blue sutures).

on the same graft in group A (test 1), while the slippage of SPEEDTRAP in group A was compared with that of Krackow suture in group B (test 2 ).

The loop end of the AM-M/-L graft was introduced through the far anteromedial portal to the femoral tunnel and fixed in the same manner, and the free ends of the graft were introduced from the joint into the tibial tunnels for the AM-M and -L grafts, respectively. Then, the graft suture from the PL graft was connected with one Double-Spike Plate (DSP; MEIRA Corp., Nagoya, Japan), whereas the 2 graft sutures for the AM-M and -L grafts were connected with 1 DSP. Finally, these grafts were fixed to the tibia with the total initial tension of $20 \mathrm{~N}$ ( $10 \mathrm{~N}$ of tension to each DSP) at $20^{\circ}$ of knee flexion.

\section{Statistical Analysis}

The Mann-Whitney $U$ test was performed to assess the difference in age, PL tunnel diameter, and crosssectional area of PL graft between group A and B. For assessment of difference in sex and meniscal injury between 2 groups, the $\chi^{2}$ test was introduced. In results, the $\chi^{2}$ test was performed to detect the significant difference in the ratio of cases with slippage in test 1 and 2, whereas the Wilcoxon signed-rank test for test 1 and Mann-Whitney $U$ test for test 2 also were used for the difference of slipped distance between SPEEDTRAP suture and Krackow stitch. A $P$ value of less than .05 was defined as a significant different. A post hoc type of power analysis with $\alpha$ of 0.05 and effect size of 0.8 showed that power of this analysis was 0.911 in test 1 and 0.762 in test 2.

\section{Results}

Thirty-eight patients were enrolled in the study (Table 1). There were 12 male and 26 female patients. Their age ranged from 15 to 59 years, with a mean age of
28 years at the time of surgery. Lateral and medial meniscal injuries were observed in 8 and 22 cases, respectively. For lateral meniscal injury, 7 cases had meniscal repair and 1 underwent meniscectomy. For the medial meniscus, 10 cases underwent meniscal repair and 1 had meniscectomy. No severe cartilage injury (more than Grade III) was found. The total number in group A and B was 20 and 18, respectively. The tunnel diameter and cross-sectional area of PL graft ${ }^{11}$ were not different between 2 groups. The slippage was observed in 16 of 20 cases $(80 \%)$ for SPEEDTRAP and in 2 of $20(10 \%)$ for Krackow suture in group A, whereas 1 case $(7 \%)$ showed slippage in group B (Fig 2). The ratio of cases with suture slippage for SPEEDTRAP was significantly larger in test 1 $(P<.001)$ and test $2(P<.001)$.

The mean slippage distance was $4.0 \pm 2.9 \mathrm{~mm}$ for SPEEDTRAP and $0.2 \pm 0.5 \mathrm{~mm}$ for Krackow stitch in group A, with a significant difference $(P<.001)$, whereas it was $0.1 \pm 0.2 \mathrm{~mm}$ for double Krackow stitch in group B, showing a significant difference from SPEEDTRAP suture $(P<.001)$.

\section{Discussion}

The principle finding of this study is that a larger amount of suture slippage was found in the SPEEDTRAP suture during graft passage through tibial tunnel compared with the Krackow suture. The Krackow stitch $^{1}$ is widely adopted to suture the graft as the secure fixation but takes a longer time to prepare graft and has a risk of needle stick injury. SPEEDTRAP was developed to save graft-preparation time, reduce graft damage, and prevent needle-stick injury, and it does not pass any sutures through the graft while tendon is tied up with sutures, based on the principle of Chinese finger trap.

Su et al. ${ }^{3}$ compared the tendon graft holding power of the MFT suture technique, which did not require needle passing through the tendon, with the Krackow stitch, the non-locking SpeedWhip (Arthrex, Naples, FL), and the locking SpeedWhip techniques. They reported the elongation of the MFT suture group was significantly smaller than that of the other techniques, whereas there was no significant difference among all 4 suture techniques with respect to ultimate failure load. Barber et al. ${ }^{9}$ compared 6 different graft suture techniques including the Krackow stitch and the SPEEDTRAP suture techniques and

Table 1. Patient Demographic Data

\begin{tabular}{lccc}
\hline & Group A & Group B & $P$ Value \\
\hline Total number & 20 & 18 & \\
Age & $26.9(15-51)$ & $28.6(15-59)$ & .942 \\
Sex, male:female & $9: 11$ & $3: 15$ & .061 \\
Meniscal injury, lateral:medial & $5: 6$ & $3: 5$ & .729 \\
Tunnel diameter (PL graft) & $4.9 \pm 0.3$ & $4.9 \pm 0.2$ & .806 \\
CSA (PL graft) & $13.4 \pm 3.3$ & $13.6 \pm 2.6$ & .740 \\
\hline \multicolumn{1}{c}{ CSA, cross sectional area; PL, posterolateral. } & &
\end{tabular}



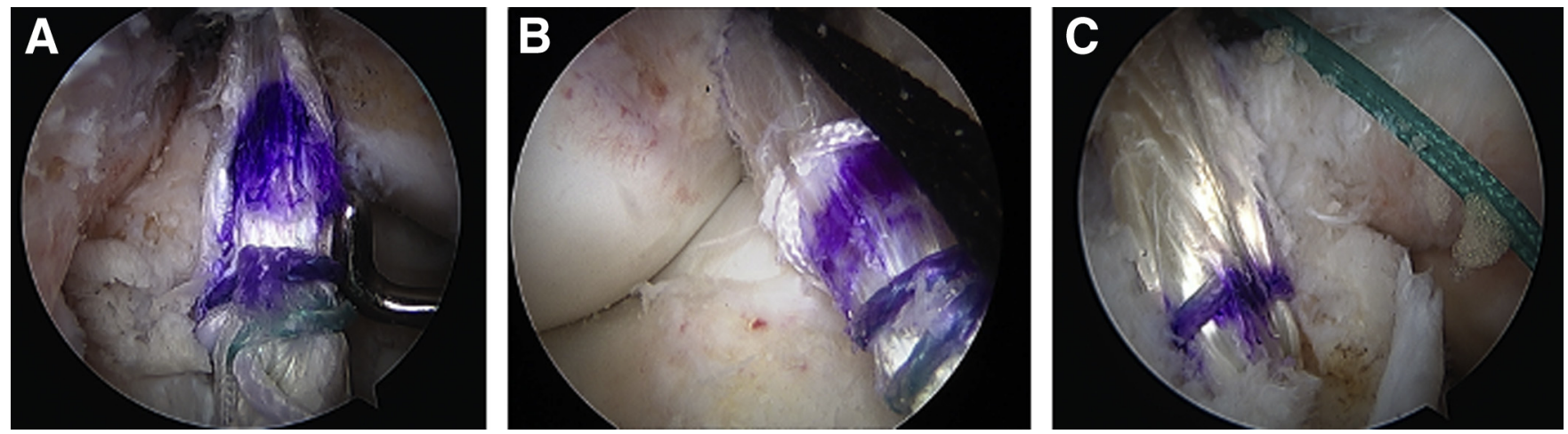

Fig 2. Arthroscopic findings. (A) Slipped SPEEDTRAP suture (green/white suture). (B) No slippage for Krackow suture (white suture) at the opposite side of SPEEDTRAP. (C) No slippage for Krackow suture in double Krackow stitch.

reported that the elongation after 500 cycles between $50 \mathrm{~N}$ and $200 \mathrm{~N}$ and the maximum failure load in SPEEDTRAP suture was equivalent to those in the Krackow stitch with FiberWire, although the elongation of SPEEDTRAP suture was larger than that of the Krackow stitch from 100 to 500 cycles. Thus, SPEEDTRAP suture may replace the Krackow stitch and can be safely used for graft preparation. However, in evaluation of needless grasping suture, most studies measured the elongation during cyclic loading and the load to failure test for the graft-suture complex. The slippage of suture on the graft during graft passage is an overlooked issue in ligament reconstruction, although it is difficult to assess this phenomenon. The current study reports the slippage of sutures passing through the tibial tunnel in ligament reconstruction.

The Chinese finger trap was originally designed as a small woven bamboo tube. When a person places their index finger in both ends of the tube and tries to pull their fingers out, the bamboo constricts and traps the fingers. SPEEDTRAP introduced this mechanism into graft suturing technique. This mechanism is strong enough to hold the graft as long as the graft-gripping suture is pulled. Then, the suture firmly grasps the graft at the greater load, while suture can slip on the graft at the lower load during cyclic loading. In the ACL reconstruction, suture from the distal end of graft is just held during the graft passage through tibial tunnel and is never kept strongly, as the graft must move into the joint. Moreover, the friction between the tibial tunnel wall and the suture on the graft can occur. Thus, decrease in the graft-holding power with SPEEDTRAP suture and friction between tunnel wall and suture could lead to suture slippage during graft passage, as no suture of SPEEDTRAP passed through the tendon. Slippage might be reduced if the suture from the graft was more strongly pulled during graft passage. In contrast, as the suture passed through the tendon in Krackow stitch, the suture seldom slipped on the graft as long as the sutures cut off the tendon.

When the length of harvested semitendinosus tendon is more than $260 \mathrm{~mm}$, we cut the tendon in half and fold them. However, in some cases, the tissue quality of the membranous portion is poor, or the gracilis tendon is quite thin and narrow. In such a case, the Chinese finger trap mechanism has an advantage for graft preparation, as the Krackow stitch has a risk to cut off the graft in pulling the graft. Thus, when SPEEDTRAP is used for graft preparation, we recommend creating a slightly larger tibial tunnel to avoid a too-snug graft passage and using a longer grip distance in SPEEDTRAP considering slippage. Furthermore, as some sutures of SPEEDTRAP stopped at the proximal end of Krackow stitch in group A, we also recommend using this device with the Krackow stitch on the opposite side to adopt the advantages of both suture techniques (Fig 3).

\section{Limitations}

There are some limitations in this study. First, friction between tunnel wall and graft can be different, as there is some variability in graft and/or tunnel size. To reduce

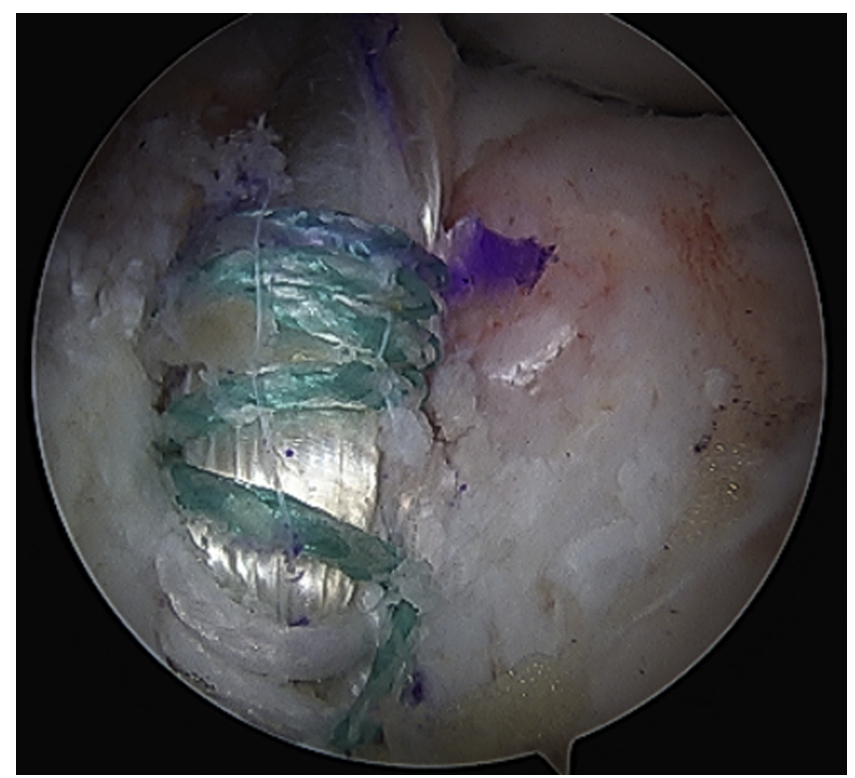

Fig 3. Slipped SPEEDTRAP sutures (green/white suture) stopped at the proximal end of Krackow suture (white suture). 
this variation, we compared both suture techniques on the same graft in group A. The second is that 2 sutures were passed for one graft. The suture on the opposite side might affect the slippage, while the possibility of graft failure increased in case of one suture for graft. Third, SPEEDTRAP suture was used only for membranous portion. The results might change in use for tendinous portion.

\section{Conclusions}

At the time of PL graft passage through the tibial tunnel in ACL reconstruction, there was significantly less slippage observed with the Krackow stitch compared with the SPEEDTRAP stitch.

\section{References}

1. Krackow KA, Thomas SC, Jones LC. A new stitch for ligament-tendon fixation. Brief note. J Bone Joint Surg Am 1986;68:764-766.

2. Deramo DM, White KL, Parks BG, Hinton RY. Krackow locking stich versus nonlocking premanufactured loop stitch for soft-tissue fixation: A biomechanical study. Arthroscopy 2008;24:599-603.

3. Su WR, Chu CH, Lin CL, Lin CJ, Jou IM, Chang CW. The modified finger-trap suture technique: A biomechanical comparison of a novel suture technique for graft fixation. Arthroscopy 2012;28:702-710.
4. Martin DK, Falworth MS. Anterior cruciate ligament graft preparation: A new and quick alternative to the whipstitch. Arthroscopy 2007;3:326.el-326.e3.

5. Masoumiganjgah A, Dhanjee U, Mwaturura T, Carmody C. Hamstrings tendon graft preparation for anterior cruciate ligament reconstruction using the WhipKnot soft tissue cinch technique. Australasian Med J 2012;5:236-238.

6. Sakaguchi K, Tachibana Y, Oda H. Biomechanical properties of porcine flexor tendon fixation with varying throws and stitch methods. Am J Sports Med 2012;40:1641-1645.

7. Wang RY, Arciero RA, Obopilwe E, Mazzocca AD. A comparison of structural and mechanical properties of tubularized and native semitendinosus graft. Am J Sports Med 2010;38:1246-1249.

8. Camarda L, Giambartino S, Lauria M, Saporito M, Triolo V, D'Arienzo M. Surgical time for graft preparation using different suture techniques. Muscles Ligaments Tendons J 2016:6:236-240.

9. Barber FA, Howard MS, Piccirillo J, Spenciner DB. A biomechanical comparison of six suture configurations for soft tissue-based graft traction and fixation. Arthroscopy 2019;35:1163-1169.

10. Shino K, Nakata K, Nakamura N, et al. Anatomic anterior cruciate ligament reconstruction using two double-looped hamstring tendon grafts via twin femoral and triple tibial tunnels. Operative Tech Orthop 2005;15:130-134.

11. Hamada M, Shino K, Mitsuoka T, Abe N, Horibe S. Crosssectional area measurement of the semitendinosus tendon for anterior cruciate ligament reconstruction. Arthroscopy 1998;14:696-701. 